\title{
Analysis of the Effect of Idle Rollers on Tension Vibration in Discontinuous Web Transport Systems
}

\author{
Jiankui Chen ${ }^{1, a}$, Yongan Huang ${ }^{2, b}$, and Zhouping Yin ${ }^{3, c}$ \\ ${ }^{1}$ Department of Control Science and Engineering, Huazhong University of Science and Technology, \\ Wuhan, 430074, P. R. China \\ ${ }^{123}$ State Key Lab of Digital Manufacturing Equipment \& Technology, Huazhong University of \\ Science and Technology, Wuhan, 430074, P. R. China \\ ajiankuichen@gmail.com, byahuang@hust.edu.cn, ${ }^{c}$ yinzhp@hust.edu.cn
}

Keywords: Tension control, idle roller, discontinuous winding, tension vibration, programmable multiple axis controller.

\begin{abstract}
Tension control and precise conveyance in web-handling machines are critical to ensure product quality. This paper is focused on analyzing the effect of idle rollers on flexible web tension vibration for discontinuous web transport systems. First, the simplified algebraic dynamical models of the inertia and friction are presented, which plays an important role for the design of idle rollers' layout systems. Second, the tension vibration test method and tension data collection scheme are introduced, respectively. Finally, based on the mathematical model and test strategies, an experiment platform is established. Several experiments have been performed to show idle rollers' effect on tension control and tension vibration in the discontinuous web transport system.
\end{abstract}

\section{Introduction}

The roll-to-roll (R2R) processing plays an increasingly important role in the high-throughput fabrication of flexible electronics, especially in discontinuous web transport systems. R2R processing could revolutionize large area electronics manufacturing [1], such as flexible display, e-textile [2], thin film solar cell [3], and large-area sensor. It is significantly important to control the tension and the speed in the web feeding, while the thickness of the web is less than 50 micrometers. A tension control system of moving web is inherently sensitive to external disturbances during operation, and the time-varying payload or torque of the driving motors can cause severe tension variation.

According to actual working conditions, the tension control system may be subjected to many uncertainties, such as a periodic disturbance from the eccentricity of the driving pulley, or a time-varying radius of the unwinding/rewinding rollers, misalignment of rollers, and nonuniform web. Current trends in the development of flexible electronics systems show that the R2R processes of web system may be the choice as an economic production method in the future. Electronic elements, like flexible batteries, labels, or Stretchable ICs [4] are etched or printed on the large-area web. These new jobsites usually need the flexible web to be transported in a discontinuous way, which brings new requirements for tension control and position control because of frequent acceleration and deceleration to the web transport system. With the changing accelerations, idle rollers start to play an important role in tension control, while ignored in continuous web transport systems. The intermittent tension fluctuation may damage the elements' electronic, magnetic or optical properties.

Due to the difficulty and importance in industry, web tension control problems have drawn the attention of many researchers. Based on theoretical analysis and experimental results, Ebler et al. [5] conclude that both dancer arm and load cell systems can be used for tension regulation. Many mathematical models of web transport system have been proposed to suit for different tension requirements. Most of them considered the effects of the external disturbances or/and uncertainties, such as the variable inertias, the lateral weave, friction and roller shape.

Shin et al. [6] introduced a mathematical model, based upon an adaptive eccentricity estimator, to reject the disturbance caused by unknown shapes of rollers. PID control, feed-forward control, feedback control, and decentralized control were solely or hybrid used in web tension/speed control 
system. Reid et al. [7] proposed PID control with the fixed gains and variable gains in the web tension system. For the variable-gain PID control, the controlled parameters are continuously adjusted based on the diameters of the unwind/rewind rollers. Chen et al. [8] presented one tension and position hybrid control method for a discontinuous web transport system, used in Radio Frequency Identification (RFID) electronic-tag integrated equipments. Wolfermann and Schroder [9] used an output feedback method to control the speed of the driven rollers. An observer was designed to decouple the drives from the web tension acting on the driven rollers and used to improve the speed control of the driven rollers. This approach leads considerable improvement to speed control of the driven rollers, but cannot avoid disturbance between web spans.

However, the mentioned works pay little attention on the study of idle rollers and tension control in the discontinuous web transport jobsite. Discontinuous convey has more strict requirements for tension vibration than continuous convey. Frequent start-stop causes biggish tension disturbance and web deformation in discontinuous web process, which encumbers the tension stableness. The application domain of discontinuous web transport system is rapidly expanding because of the increasing stringent requirement arising from developments in flexible electronic elements.

The primary goal of this paper is to analyze the effect of idle rollers on tension vibration in discontinuous web transport systems. Firstly, the mathematical model for the parameters of idle rollers, such as the inertia and the friction force, will be developed firstly. Secondly, the tension test method will be described. Finally, some experiment results are proposed to illustrate the effect of idle rollers on tension vibration and the feasibility of this tension test scheme.

\section{Mathematical Modeling}

In the discontinuous web transport system, the feed process is periodically implemented. The web velocity will also be changed periodically, as shown in Fig. 1 . The parameters, $t_{\mathrm{a}}, t_{\mathrm{c}}, t_{\mathrm{d}}$ and $t_{\mathrm{p}}$ are used to present the acceleration time, the uniform time, the deceleration time and the pause time, respectively. In different motion time, the web transport system will have diverse dynamic performance. In order to get the mathematical dynamical tension vibration model of the web system, it is supposed that there is no explicit slippage between the flexible web material and idle rollers, and the web deformation is ignored.

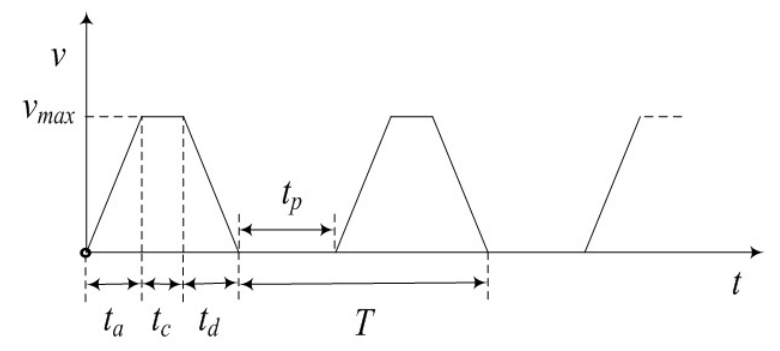

Fig. 1. Periodical change of web velocity

Effect of the Inertia. In discontinuous web transport systems, the rotation of the idle rollers is caused by the alteration of web tension. In the acceleration and deceleration time phase, flexible web drags the idle rollers to rotate or return to motionless. A cross-sectional view of the simplified idle roller is shown in Fig. 2. The associated variables for this section are radius $r$ and $r_{0}$, wrap angle $\alpha$ and tension $T_{1}, T_{2}$. 


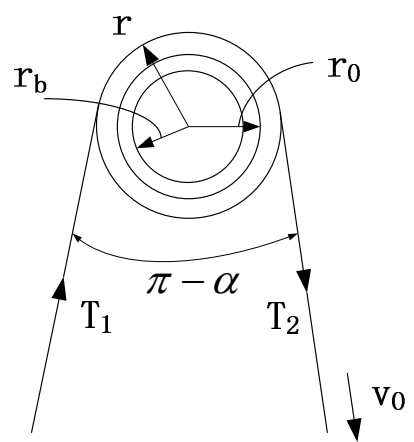

Fig. 2. Cross-sectional view of the simplified idle roller

In the uniform and pause time phase, the idle roller keeps static and the web tension $T_{1}$ is equal with $T_{2}$.

But in the acceleration and deceleration time phase, the idle roller's rotate speed is afforded by the $T_{1}$ and $T_{2}$. Ignored the viscosity friction, the relation between the web tension vibration and the inertia of the idle roller can be represented as

$$
\Delta T_{a} r=J \dot{\omega}
$$

where $\Delta T_{a}=\left|T_{2}-T_{1}\right|$ is the web tension vibration caused by the idle roller inertia, $J . \omega$ is velocity of rotation.

Synthesizing (1) gives

$$
\Delta T_{a}=\frac{\pi N L \rho\left(r^{2}-r_{0}^{2}\right)^{2}}{r^{2}} a
$$

where $L$ is the length of the idle roller, $\rho$ is density, and $a$ is rotate acceleration, $N$ is the quantity of the idle roller in the web transport system.

In this discontinuous web transport system, (2) illuminates the interaction between the constants and the variables. It can be seen that the parameters of the idle roller directly correlated with web tension vibration. When the dimensions of the idle roller is confirmed, tension vibration Is proportional to the web feeding acceleration and idle roller's quantity.

Effect of the Friction. Static friction between the flexible web and the idle roller is used to start and stop the rotation. When the static friction breaks the critical value, the web will try to slide on the outside surface of the idle roller. Analyzing this critical sliding state, shown in Fig. 3, gives

$$
\mu_{s} d N_{p}=\left(T_{\mathrm{p}}+d T_{P}\right) \cos \left(d_{c} / 2\right)-T_{\mathrm{p}} \cos \left(d_{c} / 2\right)
$$

where $d_{c}$ is tiny angle, $T_{p}$ is tension in the tiny web, $d N_{p}$ is positive pressure, $\mu_{s}$ is coefficient of friction, and $d T_{p}$ is tension vibration in $d_{c}$. 


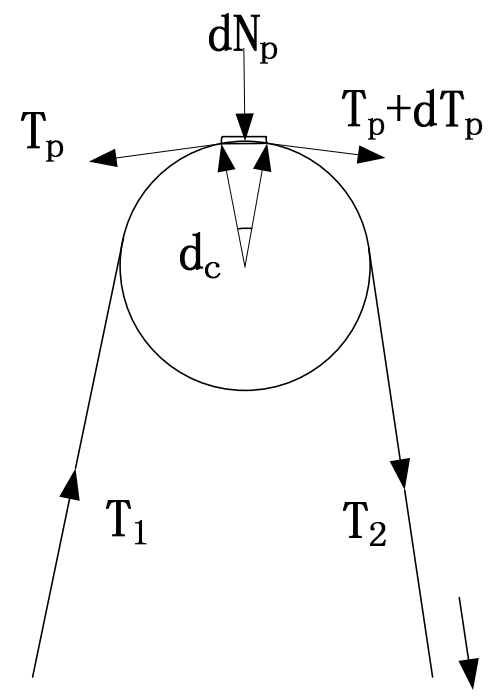

Fig. 3. Cross-sectional view of the critical sliding state

Approximately calculating, $\sin \left(d_{c} / 2\right)=d_{c} / 2, \cos \left(d_{c} / 2\right)=1$, and ignoring $d T_{p} \sin \left(d_{c} / 2\right)$, results in $d T_{P} / T_{p}=\mu_{s} d_{c}$.

In the uniformly acceleration motion state, the maximum tension vibration is limited by coulomb's static friction. That is

$$
\Delta T=\left|T_{2}-T_{1}\right|=T_{\mathrm{r}}\left(e^{\mu_{\mathrm{s}} \alpha}-1\right)
$$

where $T_{r}$ is reference tension. When $T_{r}$ is initialized and set to constant, affordable tension vibration of the idle roller can increase with the rise of $\alpha$.

Ignoring other effects of idle rollers, such as bearing frictions, in the critical sliding state, gets $\Delta T=\Delta T_{a}$. Let $T_{2}+T_{1}=2 T_{r}+\Delta T$, therefore

$$
r^{2}\left(e^{\mu_{s} \alpha}-1\right) T_{\mathrm{r}}-\pi N L \rho\left(r^{2}-r_{0}^{2}\right)^{2} a=0
$$

\section{Test Scheme}

One test scheme is proposed to monitor the tension vibration, and the layout is shown in Fig. 4. The effect of idle rollers on tension vibration can be estimated by changing the number of the idle rollers and feeding acceleration.

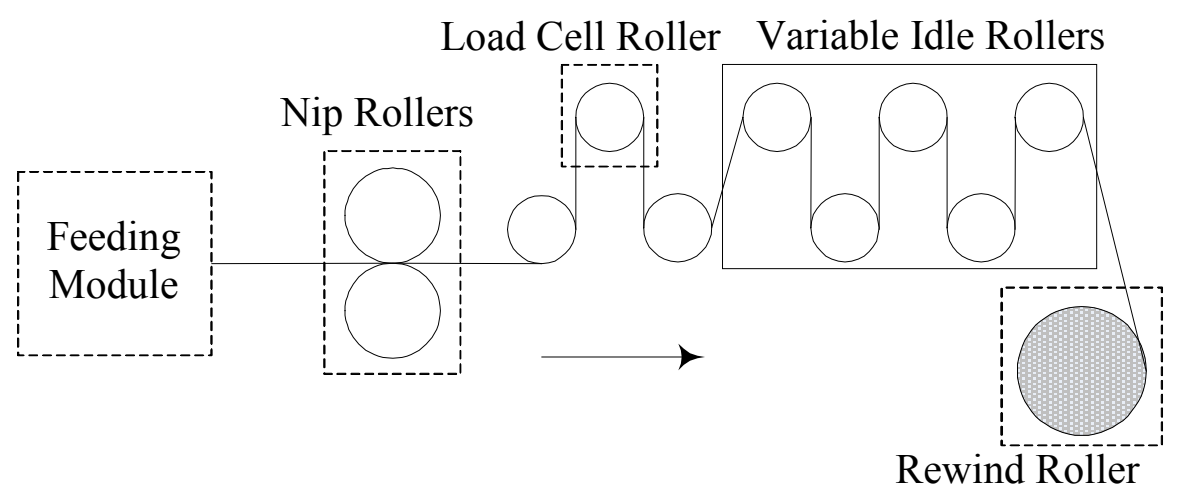

Fig. 4. Test scheme layout for tension vibration 
The block diagram of the proposed tension control and data collection scheme is shown in Fig.5. All operations for the tension control and web feeding control are completed by the Turbo PMAC (programmable multiple axis controller) board, which is provided by the Delta Tau Data Systems Inc. This card makes it possible for users to compile individualized programs to the card directly and modify the control structure. In this method, the tension data are detected from the load cell, and can be used to analyzed and disposed to control the tension, and also can be used to modify the input value at the same time. $T_{r}$ is the reference tension and $P_{r}$ is feeding web length.

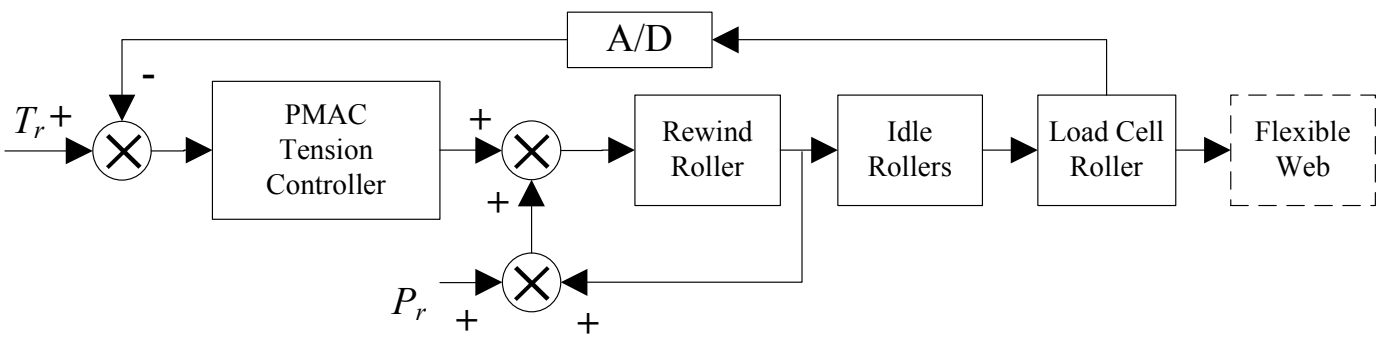

Fig. 5. Proposed tension control and data collection scheme

\section{Implementing Results}

In order to verify the effect of idle rollers on tension vibration, a large experimental web line has been built to mimic most of the features of an industrial web transport system. The R2R web transport system consists of unwind roller, rewind roller, nip rollers, load cell rollers and more than 20 idle rollers, as shown in Fig.6. Four three-phase induction motors, with 750w capacity, are used to drive web feeding rollers. Each motor is equipped with one reducer to augment the torques. The web material used in the experiments is PET (Ploy-ethylene Terephthalate). PET is considered as one of the best organic-web candidates for flexible electronics, while it features the industrial ability to be processed in a roll-to-roll fashion.

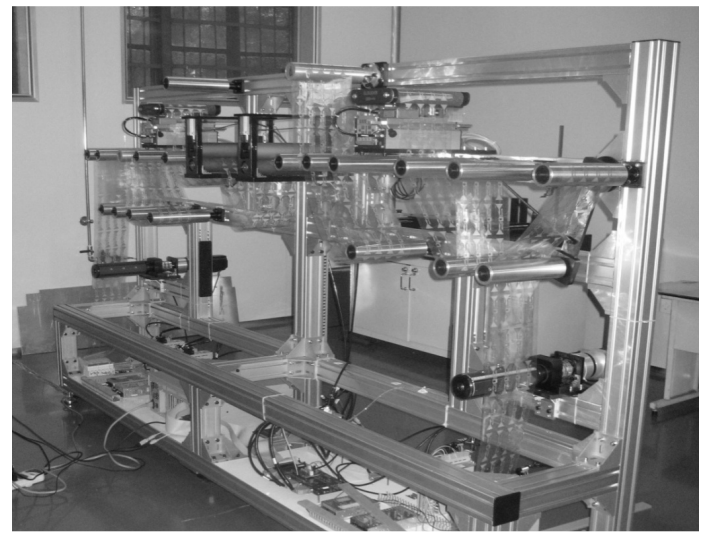

Fig. 6. R2R web transport system in a clean room environment

The properties of the web used in the experiments are: (1) Elastic modulus $400 \mathrm{~kg} / \mathrm{mm}^{2}$, (2) Width $120 \mathrm{~mm}$, (3) Thickness $40 \mu \mathrm{m}$. The comment experiment conditions are: (1) reference tension $10.2 \mathrm{~N}$, (2) web length in discontinuous periodical feeding $400 \mathrm{~mm}$, (3) tension data sampling interval is $0.005 \mathrm{~s},(4)$ Reference maximum feeding velocity $0.21 \mathrm{~m} / \mathrm{s}$.

Experiment results for the following cases are shown as follows.

Case 1): The acceleration time $t_{a}=150 \mathrm{~ms}$; the deceleration time $t_{d}=150 \mathrm{~ms}$.

Case 2): The acceleration time $t_{a}=300 \mathrm{~ms}$; the deceleration time $t_{d}=300 \mathrm{~ms}$. 
Results of the experiments are shown in Fig. 7-12. It can be seen that the tension keeps benignly steady in an acceptable confined scope except in the time of $t_{a}$ and $t_{d}$. While starting and stopping of the discontinuous web conveying, the tension is changed obviously and has some confusing oscillations. The acceleration of the web generates positive damping while deceleration generates a negative or destabilizing damping.

Fig. 7-9 shows the results for case 1). Tension vibration amplitude varies from $\pm 25 \%$ to $\pm 50 \%$ with the number of idle rollers changing from 4 to 8 . Based on equations (1)-(5), calculate the tension vibration, affected by idle rollers, and the results should be $\pm 15 \%$ to $\pm 30 \%$. Idle rollers cause biggish tension disturbance in discontinuous web process, and play important role in tension vibration.

The results for case 1) are shown in Fig. 10-12. While the feeding acceleration and deceleration is reduced, the tension vibration amplitude is evidently decreased too, $\pm 20 \%$ with 4 idle rollers, and $\pm 30 \%$ with 8 idle rollers.

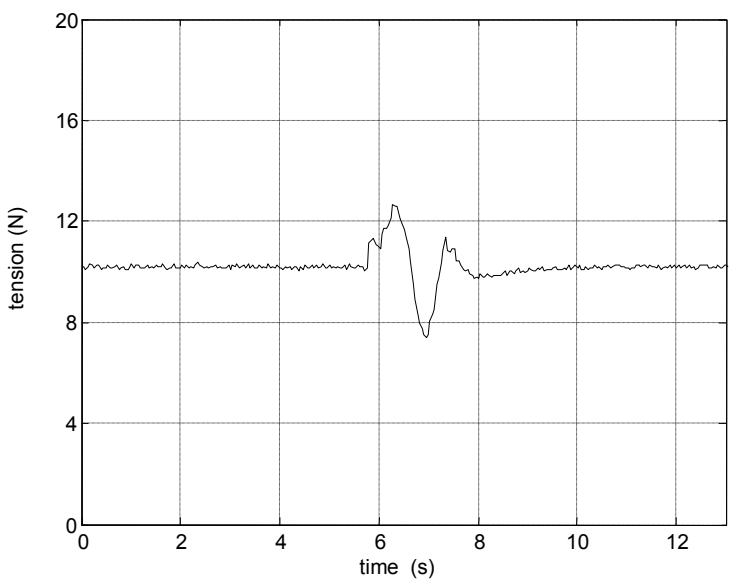

Fig. 7. 4 idle rollers in case 1)

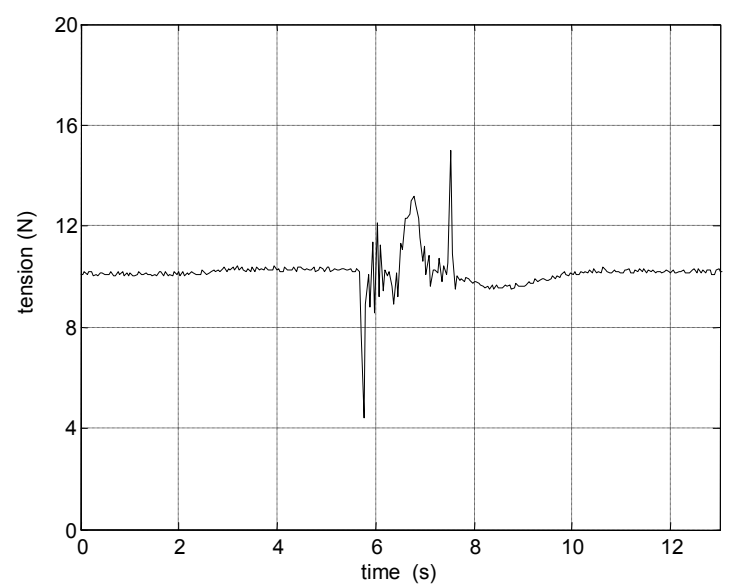

Fig. 9. 8 idle rollers in case 1)

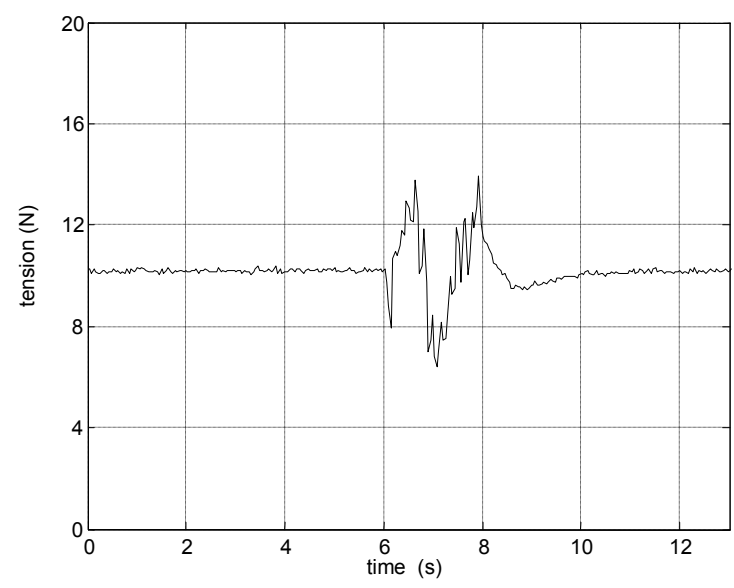

Fig. 8. 6 idle rollers in case 1)

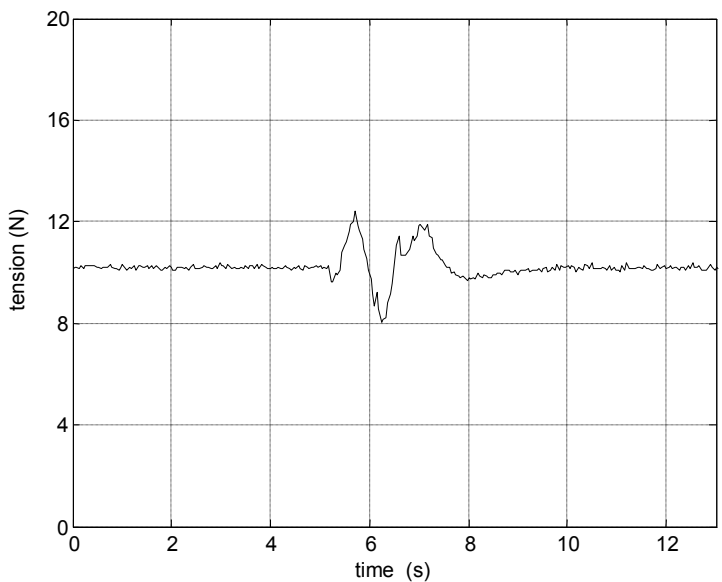

Fig. 10. 4 idle rollers in case 2) 


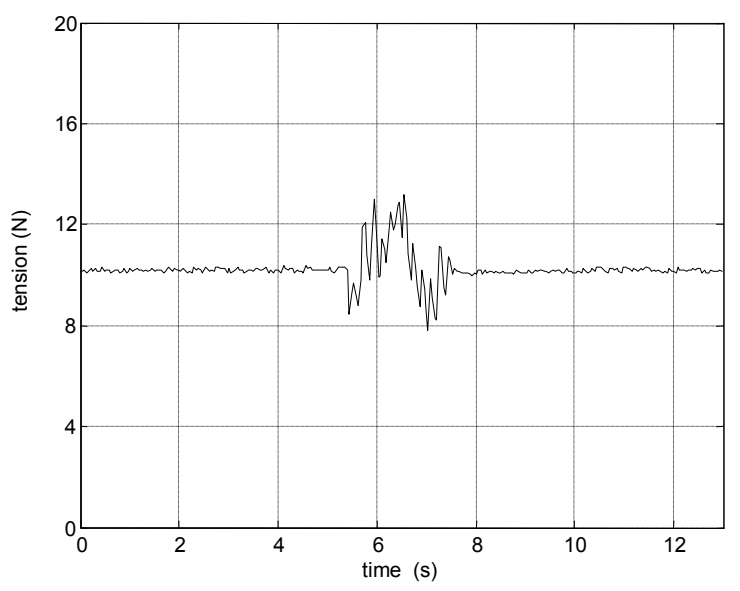

Fig. 11. 6 idle rollers in case 2)

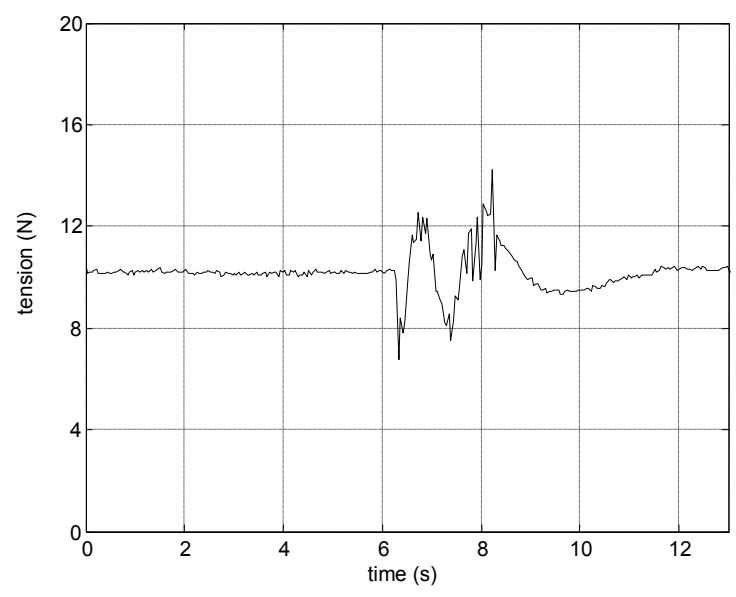

Fig. 12. 8 idle rollers in case 2)

\section{Summary}

This paper outlined the effect of idle rollers on tension control and proposed one test scheme to measure tension vibration. It is important to note that there exists biggish tension fluctuation in the starting and stopping of feeding process. The tension vibration amplitude will increase while augmenting the quantity of idle rollers and decrease with the acceleration's descending. The presented mathematical models can also be used to design the discontinuous web transport system. There is some uncertain steady error in the tension vibration. Future work will focus on solving these problems.

\section{Acknowledgment}

This work was supported by the National Technology Support Project Of China ( Project No. 2009BAF40B04), the National Fundamental Research Program (973) Of China (Project No. 2009CB724204) .

\section{References}

[1] S. Ahn and L. Guo: High-speed roll-to-roll nanoimprint lithography on flexible plastic substrates, Adv. Mater., vol. 20, no. 11, pp. 2044-2049, Jun. 2008.

[2] Y. Kim, H. Kim and H. Yoo: Electrical characterization of screen-printed circuits on the fabric, IEEE Trans. Adv. Packag., vol.33, no. 1, pp. 196-205, Apr. 2006.

[3] A. John, T. Someya and Y. Huang: Materials and mechanics for stretchable electronics. Science, vol. 327, no. 5973, pp. 1603-1607, Mar. 2010.

[4] A. Mayer, S. Scully, B. Hardin, M. Rowell and M. McGehee: Polymer-based solar cells. Mater. Today, vol. 10, no. 11, pp. 28-33, Nov. 2007.

[5] N. Elber, R. Arnason, G. Michaelis, and N. D'Sa: Tension control: Dancer rolls or load cells, IEEE Trans. Ind. Appl., vol. 29, no. 4, pp.727-739, Jul./Aug.1993.

[6] K. Shin, J. Jang, H. Kang, and S. Song: Compensation Method for Tension Disturbance Due to an Unknown Roll Shape in a Web Transport System, IEEE Trans. Ind. Appl., vol. 39, no. 5, pp. 1422-1428, Sep./Oct. 2003.

[7] K. Reid, K. Shin, and K. Lin: Variable-Gain Control of Longitudinal Tension in A Web Transport System, ASME Web Handling, pp. 87-100, 1992.

[8] J. Chen, Z. Yin, Y. Xiong and J. Quan: A hybrid control method of tension and position for a discontinuous web transport system, IEEE International Conference on Information and Automation, pp. 265-270, 2009.

[9] W. Wolfermann and D. Schroder: New Decentralized Control in Processing Machines with Continuous Moving Webs, Processing of the Third International Conference on Web Handling, pp.96-115, 1995. 